\title{
String theories as the adiabatic limit of Yang-Mills theory
}

\author{
Alexander D. Popov* \\ Institut für Theoretische Physik, Leibniz Universität Hannover, Appelstraße 2, 30167 Hannover, Germany
}

(Received 4 June 2015; published 4 August 2015)

\begin{abstract}
We consider Yang-Mills theory with a matrix gauge group $G$ on a direct product manifold $M=\Sigma_{2} \times H^{2}$, where $\Sigma_{2}$ is a two-dimensional Lorentzian manifold and $H^{2}$ is a two-dimensional open disc with the boundary $S^{1}=\partial H^{2}$. The Euler-Lagrange equations for the metric on $\Sigma_{2}$ yield constraint equations for the Yang-Mills energy-momentum tensor. We show that in the adiabatic limit, when the metric on $H^{2}$ is scaled down, the Yang-Mills equations plus constraints on the energy-momentum tensor become the equations describing strings with a world sheet $\Sigma_{2}$ moving in the based loop group $\Omega G=C^{\infty}\left(S^{1}, G\right) / G$, where $S^{1}$ is the boundary of $H^{2}$. By choosing $G=\mathbb{R}^{d-1,1}$ and putting to zero all parameters in $\Omega \mathbb{R}^{d-1,1}$ besides $\mathbb{R}^{d-1,1}$, we get a string moving in $\mathbb{R}^{d-1,1}$. In another paper of the author, it was described how one can obtain the Green-Schwarz superstring action from Yang-Mills theory on $\Sigma_{2} \times H^{2}$ while $H^{2}$ shrinks to a point. Here we also consider Yang-Mills theory on a three-dimensional manifold $\Sigma_{2} \times S^{1}$ and show that in the limit when the radius of $S^{1}$ tends to zero, the Yang-Mills action functional supplemented by a Wess-Zumino-type term becomes the Green-Schwarz superstring action.
\end{abstract}

DOI: 10.1103/PhysRevD.92.045003

PACS numbers: 11.15.-q, 11.15.Kc, 11.25.-w

\section{INTRODUCTION}

Superstring theory has a long history [1-3] and pretends on description of all four known forces in nature. In the low-energy limit superstring theories describe supergravity in ten dimensions or supergravity interacting with supersymmetric Yang-Mills (SYM) theory. On the other hand, Yang-Mills and SYM theories in four dimensions give descriptions of three main forces in nature not including gravity [4-7]. The aim of this short paper is to show that bosonic strings (both open and closed) as well as type I, IIA, and IIB superstrings can be obtained as a subsector of pure Yang-Mills theory with some constraints on the YangMills energy-momentum tensor. Put differently, knowing the action for superstrings with a world sheet $\Sigma_{2}$, we introduce a Yang-Mills action functional on $\Sigma_{2} \times H^{2}$ or on $\Sigma_{2} \times S^{1}$ such that the Yang-Mills action becomes the Green-Schwarz superstring action while $H^{2}$ or $S^{1}$ shrink to a point. We will work in Lorentzian signature, but all calculations can be repeated for the Euclidean signature of spacetime.

\section{YANG-MILLS EQUATIONS}

Consider Yang-Mills theory with a matrix gauge group $G$ on a direct product manifold $M=\Sigma_{2} \times H^{2}$, where $\Sigma_{2}$ is a two-dimensional Lorentzian manifold (flat case is included) with local coordinates $x^{a}, a, b, \ldots=1,2$, and a metric tensor $g_{\Sigma_{2}}=\left(g_{a b}\right), H^{2}$ is the disc with coordinates $x^{i}, i, j, \ldots=3,4$, satisfying the inequality $\left(x^{3}\right)^{2}+\left(x^{4}\right)^{2}<1$, and the metric $g_{H^{2}}=\left(g_{i j}\right)$. Then $\left(x^{\mu}\right)=\left(x^{a}, x^{i}\right)$ are local coordinates on $M$ with $\mu=1, \ldots, 4$.

*popov@itp.uni-hannover.de
We start with the gauge potential $\mathcal{A}=\mathcal{A}_{\mu} \mathrm{d} x^{\mu}$ with values in the Lie algebra $\mathfrak{g}=\operatorname{Lie} G$ having scalar product $(\cdot, \cdot)$ defined either via trace Tr or, for Abelian groups like $\mathbb{R}^{p, q}$, $T^{p, q}$ etc., via a metric on vector spaces. The gauge field $\mathcal{F}=\mathrm{d} \mathcal{A}+\mathcal{A} \wedge \mathcal{A}$ is the $\mathfrak{g}$-valued 2-form

$$
\begin{aligned}
\mathcal{F} & =\frac{1}{2} \mathcal{F}_{\mu \nu} \mathrm{d} x^{\mu} \wedge \mathrm{d} x^{\nu} \quad \text { with } \\
\mathcal{F}_{\mu \nu} & =\partial_{\mu} \mathcal{A}_{\nu}-\partial_{\nu} \mathcal{A}_{\mu}+\left[\mathcal{A}_{\mu}, \mathcal{A}_{\nu}\right]
\end{aligned}
$$

The Yang-Mills equations on $M$ with the metric

$$
\mathrm{d} s^{2}=g_{\mu \nu} \mathrm{d} x^{\mu} \mathrm{d} x^{\nu}=g_{a b} \mathrm{~d} x^{a} \mathrm{~d} x^{b}+g_{i j} \mathrm{~d} x^{i} \mathrm{~d} x^{j}
$$

have the form

$$
D_{\mu} \mathcal{F}^{\mu \nu}:=\frac{1}{\sqrt{|\operatorname{det} g|}} \partial_{\mu}\left(\sqrt{|\operatorname{det} g|} \mathcal{F}^{\mu \nu}\right)+\left[\mathcal{A}_{\mu}, \mathcal{F}^{\mu \nu}\right]=0
$$

where $g=\left(g_{\mu \nu}\right)$ and $\partial_{\mu}=\partial / \partial x^{\mu}$.

Equations (3) follow from the standard Yang-Mills action on $M$,

$$
S=\frac{1}{4} \int_{M} \mathrm{~d}^{4} x \sqrt{\mid \operatorname{det} g} \mid\left(\mathcal{F}_{\mu \nu}, \mathcal{F}^{\mu \nu}\right),
$$

where $(\cdot, \cdot)$ is the scalar product on the Lie algebra $\mathfrak{g}$. Note that the metric $g_{\Sigma_{2}}$ on $\Sigma_{2}$ is not fixed and the EulerLagrange equations for $g_{\Sigma_{2}}$ yield the constraint equations

$$
T_{a b}=g^{\lambda \sigma}\left(\mathcal{F}_{a \lambda}, \mathcal{F}_{b \sigma}\right)-\frac{1}{4} g_{a b}\left(\mathcal{F}_{\mu \nu}, \mathcal{F}^{\mu \nu}\right)=0
$$


for the Yang-Mills energy-momentum tensor $T_{\mu \nu}$; i.e., its components along $\Sigma_{2}$ are vanishing. Note that these constraints can be satisfied for many gauge configurations; e.g., for self-dual gauge fields, not only does $T_{a b}=0$ but even $T_{\mu \nu}=0$.

\section{ADIABATIC LIMIT}

On $M=\Sigma_{2} \times H^{2}$ we have the obvious splitting

$$
\begin{gathered}
\mathcal{A}=\mathcal{A}_{\mu} \mathrm{d} x^{\mu}=\mathcal{A}_{a} \mathrm{~d} x^{a}+\mathcal{A}_{i} \mathrm{~d} x^{i} \\
\mathcal{F}=\frac{1}{2} \mathcal{F}_{\mu \nu} \mathrm{d} x^{\mu} \wedge \mathrm{d} x^{\nu}=\frac{1}{2} \mathcal{F}_{a b} \mathrm{~d} x^{a} \wedge \mathrm{d} x^{b}+\mathcal{F}_{a i} \mathrm{~d} x^{a} \wedge \mathrm{d} x^{i} \\
+\frac{1}{2} \mathcal{F}_{i j} \mathrm{~d} x^{i} \wedge \mathrm{d} x^{j}, \\
T=T_{\mu \nu} \mathrm{d} x^{\mu} \mathrm{d} x^{\nu}=T_{a b} \mathrm{~d} x^{a} \mathrm{~d} x^{b}+2 T_{a i} \mathrm{~d} x^{a} \mathrm{~d} x^{i}+T_{i j} \mathrm{~d} x^{i} \mathrm{~d} x^{j} .
\end{gathered}
$$

By using the adiabatic approach in the form presented in $[8,9]$, we deform the metric (2) and introduce the metric

$$
\mathrm{d} s_{\varepsilon}^{2}=g_{a b} \mathrm{~d} x^{a} \mathrm{~d} x^{b}+\varepsilon^{2} g_{i j} \mathrm{~d} x^{i} \mathrm{~d} x^{j},
$$

where $\varepsilon \in[0,1]$ is a real parameter. It is assumed that the fields $\mathcal{A}_{\mu}$ and $\mathcal{F}_{\mu \nu}$ smoothly depend in $\varepsilon^{2}$, i.e., $\mathcal{A}_{\mu}=$ $\mathcal{A}_{\mu}^{(0)}+\varepsilon^{2} \mathcal{A}_{\mu}^{(1)}+\cdots \quad$ and $\quad \mathcal{F}_{\mu \nu}=\mathcal{F}_{\mu \nu}^{(0)}+\varepsilon^{2} \mathcal{F}_{\mu \nu}^{(1)}+\cdots$ Furthermore, we have $\operatorname{det} g_{\varepsilon}=\varepsilon^{4} \operatorname{det}\left(g_{a b}\right) \operatorname{det}\left(g_{i j}\right)$ and

$$
\begin{aligned}
\mathcal{F}_{\varepsilon}^{a b} & =g_{\varepsilon}^{a c} g_{\varepsilon}^{b d} \mathcal{F}_{c d}=\mathcal{F}^{a b}, \\
\mathcal{F}_{\varepsilon}^{a i} & =g_{\varepsilon}^{a c} g_{\varepsilon}^{i j} \mathcal{F}_{c j}=\varepsilon^{-2} \mathcal{F}^{a i} \quad \text { and } \\
\mathcal{F}_{\varepsilon}^{i j} & =g_{\varepsilon}^{i k} g_{\varepsilon}^{j l} \mathcal{F}_{k l}=\varepsilon^{-4} \mathcal{F}^{i j},
\end{aligned}
$$

where indices in $\mathcal{F}^{\mu \nu}$ are raised by the nondeformed metric tensor $g^{\mu \nu}$.

For the deformed metric (9) the action functional (4) is changed to

$$
\begin{aligned}
S_{\varepsilon}= & \frac{1}{4} \int_{M} \mathrm{~d}^{4} x \sqrt{\left|\operatorname{det} g_{\Sigma_{2}}\right|} \sqrt{\operatorname{det} g_{H_{2}}}\left\{\varepsilon^{2}\left(\mathcal{F}_{a b}, \mathcal{F}^{a b}\right)\right. \\
& \left.+2\left(\mathcal{F}_{a i}, \mathcal{F}^{a i}\right)+\varepsilon^{-2}\left(\mathcal{F}_{i j}, \mathcal{F}^{i j}\right)\right\} .
\end{aligned}
$$

The term $\varepsilon^{-2}\left(\mathcal{F}_{i j}, \mathcal{F}^{i j}\right)$ in the Yang-Mills Lagrangian (11) diverges when $\varepsilon \rightarrow 0$. To avoid this we impose the flatness condition

$$
\mathcal{F}_{i j}^{(0)}=0 \Rightarrow \lim _{\varepsilon \rightarrow 0}\left(\varepsilon^{-1} \mathcal{F}_{i j}\right)=0
$$

on the components of the field tensor along $H^{2}$. Here $\mathcal{F}_{i j}^{(0)}=0$, but $\mathcal{F}_{i j}^{(1)}$ etc. in the $\varepsilon^{2}$ expansion must not be zero. For the deformed metric (9), the Yang-Mills equations have the form

$$
\begin{gathered}
\varepsilon^{2} D_{a} \mathcal{F}^{a b}+D_{i} \mathcal{F}^{i b}=0, \\
\varepsilon D_{a} \mathcal{F}^{a j}+\varepsilon^{-1} D_{i} \mathcal{F}^{i j}=0 .
\end{gathered}
$$

In the deformed metric (9) the constraint equations (5) become

$$
\begin{aligned}
T_{a b}^{\varepsilon}= & \varepsilon^{2}\left\{g^{c d}\left(\mathcal{F}_{a c}, \mathcal{F}_{b d}\right)-\frac{1}{4} g_{a b}\left(\mathcal{F}_{c d}, \mathcal{F}^{c d}\right)\right\} \\
& +g^{i j}\left(\mathcal{F}_{a i}, \mathcal{F}_{b j}\right)-\frac{1}{2} g_{a b}\left(\mathcal{F}_{c i}, \mathcal{F}^{c i}\right) \\
& -\frac{1}{4} \varepsilon^{-2} g_{a b}\left(\mathcal{F}_{i j}, \mathcal{F}^{i j}\right)=0 .
\end{aligned}
$$

In the adiabatic limit $\varepsilon \rightarrow 0$, the Yang-Mills equations (13) and (14) become

$$
\begin{aligned}
& D_{i} \mathcal{F}^{i b}=0, \\
& D_{a} \mathcal{F}^{a j}=0,
\end{aligned}
$$

since the $\varepsilon^{-1}$ term vanishes due to (12). We also keep (17) since it follows from the action (11) after taking the limit $\varepsilon \rightarrow 0$. One can see that the constraint equations (15) are nonsingular in the limit $\varepsilon \rightarrow 0$ also due to (12):

$$
T_{a b}^{0}=g^{i j}\left(\mathcal{F}_{a i}, \mathcal{F}_{b j}\right)-\frac{1}{2} g_{a b}\left(\mathcal{F}_{c i}, \mathcal{F}^{c i}\right)=0 .
$$

Note that for the adiabatic limit of instanton equations [8,9] the constraints (15) disappear since the energy-momentum tensor for self-dual and anti-self-dual gauge fields vanishes on any four-manifold $M$.

\section{FLAT CONNECTIONS}

Now we start to consider the flatness equation (12), the equations (16), (17), and the constraint equations (18). From now on we will consider only zero modes in $\varepsilon^{2}$ expansions and equations on them. For simplicity of notation we will omit the index "(0)" from all $\mathcal{A}^{(0)}$ and $\mathcal{F}^{(0)}$ tensor components. In the adiabatic approach it is assumed that all fields depend on coordinates $x^{a} \in \Sigma_{2}$ only via moduli parameters $\phi^{\alpha}\left(x^{a}\right), \alpha, \beta=1,2, \ldots$, appearing in the solutions of the flatness equation (12).

Flat connection $\mathcal{A}_{H^{2}}:=\mathcal{A}_{i} \mathrm{~d} x^{i}$ on $H^{2}$ has the form

$$
\mathcal{A}_{H^{2}}=g^{-1} \hat{\mathrm{d}} g \quad \text { with } \quad \hat{\mathrm{d}}=\mathrm{d} x^{i} \partial_{i} \quad \text { for } \partial_{i}=\frac{\partial}{\partial x^{i}},
$$

where $g=g\left(\phi^{\alpha}\left(x^{a}\right), x^{i}\right)$ is a smooth map from $H^{2}$ into the gauge group $G$ for any fixed $x^{a} \in \Sigma_{2}$.

Let us introduce on $H^{2}$ spherical coordinates: $x^{3}=$ $\rho \cos \varphi$ and $x^{4}=\rho \sin \varphi$. Using these coordinates, we impose on $g$ the condition $g\left(\varphi=0, \rho^{2} \rightarrow 1\right)=\mathrm{Id}$ (framing) and denote by $C_{0}^{\infty}\left(H^{2}, G\right)$ the space of framed 
flat connections on $H^{2}$ given by (19). On $H^{2}$, as on a manifold with a boundary, the group of gauge transformations for any fixed $x^{a} \in \Sigma_{2}$ is defined as (see e.g., [9-11])

$$
\mathcal{G}_{H^{2}}=\left\{g: H^{2} \rightarrow G \mid g \rightarrow \text { Id } \quad \text { for } \rho^{2} \rightarrow 1\right\} .
$$

Hence the solution space of the equation (12) is the infinite-dimensional group $\mathcal{N}=C_{0}^{\infty}\left(H^{2}, G\right)$, and the moduli space of solutions is the based loop group $[9,10,12]$

$$
\mathcal{M}=C_{0}^{\infty}\left(H^{2}, G\right) / \mathcal{G}_{H^{2}}=\Omega G .
$$

This space can also be represented as $\Omega G=L G / G$, where $L G=C^{\infty}\left(S^{1}, G\right)$ is the loop group with the circle $S^{1}=\partial H^{2}$ parametrized by $e^{\mathrm{i} \varphi}$.

\section{MODULI SPACE}

On the group manifold (21) we introduce local coordinates $\phi^{\alpha}$ with $\alpha=1,2, \ldots$ and recall that $\mathcal{A}_{\mu}$ 's depend on $x^{a} \in \Sigma_{2}$ only via moduli parameters $\phi^{\alpha}=\phi^{\alpha}\left(x^{a}\right)$. Then moduli of gauge fields define a map

$$
\phi: \Sigma_{2} \rightarrow \mathcal{M} \text { with } \phi\left(x^{a}\right)=\left\{\phi^{\alpha}\left(x^{a}\right)\right\} .
$$

These maps are constrained by Eqs. (16), (17), and (18). Since $\mathcal{A}_{H^{2}}$ is a flat connection for any $x^{a} \in \Sigma_{2}$, the derivatives $\partial_{a} \mathcal{A}_{i}$ have to satisfy the linearized (around $\mathcal{A}_{H^{2}}$ ) flatness condition; i.e., $\partial_{a} \mathcal{A}_{i}$ belong to the tangent space $T_{\mathcal{A}} \mathcal{N}$ of the space $\mathcal{N}=C_{0}^{\infty}\left(H^{2}, G\right)$ of framed flat connections on $H^{2}$. Using the projection $\pi: \mathcal{N} \rightarrow \mathcal{M}$ from $\mathcal{N}$ to the moduli space $\mathcal{M}$, one can decompose $\partial_{a} \mathcal{A}_{i}$ into the two parts

$$
T_{\mathcal{A}} \mathcal{N}=\pi^{*} T_{\mathcal{A}} \mathcal{M} \oplus T_{\mathcal{A}} \mathcal{G} \Leftrightarrow \partial_{a} \mathcal{A}_{i}=\left(\partial_{a} \phi^{\beta}\right) \xi_{\beta i}+D_{i} \epsilon_{a},
$$

where $\mathcal{G}$ is the gauge group $\mathcal{G}_{H^{2}}$ for any fixed $x^{a} \in \Sigma_{2}$, $\left\{\xi_{\alpha}=\xi_{\alpha i} \mathrm{~d} x^{i}\right\}$ is a local basis of tangent vectors at $T_{\mathcal{A}} \mathcal{M}$ (they form the loop Lie algebra $\Omega \mathfrak{g}$ ) and $\epsilon_{a}$ are $\mathfrak{g}$-valued gauge parameters $\left(D_{i} \epsilon_{a} \in T_{\mathcal{A}} \mathcal{G}\right)$ which are determined by the gauge-fixing conditions

$$
g^{i j} D_{i} \xi_{\alpha j}=0 \Leftrightarrow g^{i j} D_{i} D_{j} \epsilon_{a}=g^{i j} D_{i} \partial_{a} \mathcal{A}_{j} .
$$

Note also that since $\mathcal{A}_{i}\left(\phi^{\alpha}, x^{j}\right)$ depends on $x^{a}$ only via $\phi^{\alpha}$, we have

$$
\partial_{a} \mathcal{A}_{i}=\frac{\partial \mathcal{A}_{i}}{\partial \phi^{\beta}} \partial_{a} \phi^{\beta} \stackrel{(24)}{\Rightarrow} \epsilon_{a}=\left(\partial_{a} \phi^{\beta}\right) \epsilon_{\beta},
$$

where the gauge parameters $\epsilon_{\beta}$ are found by solving the equations

$$
g^{i j} D_{i} D_{j} \epsilon_{\beta}=g^{i j} D_{i} \frac{\partial \mathcal{A}_{j}}{\partial \phi^{\beta}} .
$$

Recall that $\mathcal{A}_{i}$ are given explicitly by (19) and $\mathcal{A}_{a}$ are yet free. It is natural to choose $\mathcal{A}_{a}=\epsilon_{a}[5,6]$ and obtain

$$
\mathcal{F}_{a i}=\partial_{a} \mathcal{A}_{i}-D_{i} \mathcal{A}_{a}=\left(\partial_{a} \phi^{\beta}\right) \xi_{\beta i}=\pi_{*} \partial_{a} \mathcal{A}_{i} \in T_{\mathcal{A}} \mathcal{M} .
$$

Thus, if we know the dependence of $\phi^{\alpha}$ on $x^{a}$, then we can construct

$$
\left(\mathcal{A}_{\mu}\right)=\left(\mathcal{A}_{a}, \mathcal{A}_{i}\right)=\left(\left(\partial_{a} \phi^{\beta}\right) \epsilon_{\beta}, g^{-1}\left(\phi^{\alpha}, x^{j}\right) \partial_{i} g\left(\phi^{\beta}, x^{k}\right)\right),
$$

which are in fact the components $\mathcal{A}_{\mu}^{(0)}=\mathcal{A}_{\mu}(\varepsilon=0)$.

\section{EFFECTIVE ACTION}

For finding equations for $\phi^{\alpha}\left(x^{a}\right)$, we substitute (27) into (16) and see that (16) are resolved due to (24). Substituting (27) into (17), we obtain the equations

$$
\begin{aligned}
& \frac{1}{\sqrt{\left|\operatorname{det} g_{\Sigma_{2}}\right|}} \partial_{a}\left(\sqrt{\left|\operatorname{det} g_{\Sigma_{2}}\right|} g^{a b} \partial_{b} \phi^{\beta}\right) g^{i j} \xi_{\beta j} \\
& \quad+g^{a b} g^{i j}\left(D_{a} \xi_{\beta j}\right) \partial_{b} \phi^{\beta}=0 .
\end{aligned}
$$

We should project (29) on the moduli space $\mathcal{M}=\Omega G$, metric $\mathbb{G}=\left(G_{\alpha \beta}\right)$, which is defined as

$$
G_{\alpha \beta}=\left\langle\xi_{\alpha}, \xi_{\beta}\right\rangle=\int_{H^{2}} d \operatorname{vol} g^{i j}\left(\xi_{\alpha i}, \xi_{\beta j}\right) .
$$

The projection is provided by multiplying (29) by $\left\langle\xi_{\alpha}, \cdot\right\rangle$ (cf., e.g., $[13,14])$. We obtain

$$
\begin{aligned}
& \frac{1}{\sqrt{\left|\operatorname{det} g_{\Sigma_{2}}\right|}} \partial_{a}\left(\sqrt{\left|\operatorname{det} g_{\Sigma_{2}}\right|} g^{a b} \partial_{b} \phi^{\beta}\right)\left\langle\xi_{\alpha}, \xi_{\beta}\right\rangle+g^{a b}\left\langle\xi_{\alpha}, D_{a} \xi_{\beta}\right\rangle \partial_{b} \phi^{\beta} \\
& =\frac{1}{\sqrt{\mid \operatorname{det} g_{\Sigma_{2} \mid}}} \partial_{a}\left(\sqrt{\left|\operatorname{det} g_{\Sigma_{2}}\right|} g^{a b} \partial_{b} \phi^{\beta}\right) G_{\alpha \beta}+\left\langle\xi_{\alpha}, \nabla_{\gamma} \xi_{\beta}\right\rangle g^{a b} \partial_{a} \phi^{\gamma} \partial_{b} \phi^{\beta} \\
& =G_{\alpha \sigma}\left\{\frac{1}{\sqrt{\left|\operatorname{det} g_{\Sigma_{2}}\right|}} \partial_{a}\left(\sqrt{\mid \operatorname{det} g_{\Sigma_{2}}} \mid g^{a b} \partial_{b} \phi^{\sigma}\right)+\Gamma_{\beta \gamma}^{\sigma} g^{a b} \partial_{a} \phi^{\beta} \partial_{b} \phi^{\gamma}\right\}=0,
\end{aligned}
$$


where

$$
\Gamma_{\beta \gamma}^{\sigma}=\frac{1}{2} G^{\sigma \lambda}\left(\partial_{\gamma} G_{\beta \lambda}+\partial_{\beta} G_{\gamma \lambda}-\partial_{\lambda} G_{\beta \gamma}\right) \quad \text { with } \quad \partial_{\gamma}:=\frac{\partial}{\partial \phi^{\gamma}}
$$

are the Christoffel symbols and $\nabla_{\gamma}$ are the corresponding covariant derivatives on the moduli space $\mathcal{M}$ of flat connections on $H^{2}$.

The equations

$$
\begin{aligned}
& \frac{1}{\sqrt{\left|\operatorname{det} g_{\Sigma_{2}}\right|}} \partial_{a}\left(\sqrt{\left|\operatorname{det} g_{\Sigma_{2}}\right|} g^{a b} \partial_{b} \phi^{\alpha}\right) \\
& +\Gamma_{\beta \gamma}^{\alpha} g^{a b} \partial_{a} \phi^{\beta} \partial_{b} \phi^{\gamma}=0
\end{aligned}
$$

are the Euler-Lagrange equations for the effective action

$$
S_{\text {eff }}=\int_{\Sigma_{2}} \mathrm{~d} x^{1} \mathrm{~d} x^{2} \sqrt{-\operatorname{det}\left(g_{a b}\right)} g^{c d} G_{\alpha \beta} \partial_{c} \phi^{\alpha} \partial_{d} \phi^{\beta}
$$

obtained from the action functional (11) in the adiabatic limit $\varepsilon \rightarrow 0$; it appears from the term $\left(\mathcal{F}_{a i}, \mathcal{F}^{a i}\right)$ in (11) (other terms vanish). Equations (33) are the standard sigmamodel equations defining maps from $\Sigma_{2}$ into the based loop group $\Omega G$.

\section{VIRASORO CONSTRAINTS}

The last undiscussed equations are the constraints (18). Substituting (27) into (18), we obtain

$g^{i j}\left(\xi_{\alpha i}, \xi_{\beta j}\right) \partial_{a} \phi^{\alpha} \partial_{b} \phi^{\beta}-\frac{1}{2} g_{a b} g^{c d} g^{i j}\left(\xi_{\alpha i}, \xi_{\beta j}\right) \partial_{c} \phi^{\alpha} \partial_{d} \phi^{\beta}=0$.

Integrating (35) over $H^{2}$ (projection on $\mathcal{M}$ ), we get

$$
G_{\alpha \beta} \partial_{a} \phi^{\alpha} \partial_{b} \phi^{\beta}-\frac{1}{2} g_{a b} g^{c d} G_{\alpha \beta} \partial_{c} \phi^{\alpha} \partial_{d} \phi^{\beta}=0 .
$$

These are equations which one will obtain from (34) by varying with respect to $g_{a b}$. Thus,

$$
T_{a b}^{V}=G_{\alpha \beta} \partial_{a} \phi^{\alpha} \partial_{b} \phi^{\beta}-\frac{1}{2} g_{a b} g^{c d} G_{\alpha \beta} \partial_{c} \phi^{\alpha} \partial_{d} \phi^{\beta}
$$

is the traceless stress-energy tensor and Eqs. (36) are the Virasoro constraints accompanying the Polyakov string action (34).

\section{B FIELD}

In string theory the action (34) is often extended by adding the $B$-field term. This term can be obtained from the topological Yang-Mills term $\frac{1}{2} \int_{M} \mathrm{~d}^{4} x \sqrt{\operatorname{det} g_{H_{2}}^{\varepsilon}} \varepsilon_{\mu \nu \lambda \sigma}\left(\mathcal{F}_{\varepsilon}^{\mu \nu}, \mathcal{F}_{\varepsilon}^{\lambda \sigma}\right)$,

which in the adiabatic limit $\varepsilon \rightarrow 0$ becomes

$$
\begin{gathered}
\int_{M} \mathrm{~d}^{4} x \sqrt{\operatorname{det} g_{H_{2}}} \varepsilon^{a b} \varepsilon^{i j}\left(\mathcal{F}_{a i}, \mathcal{F}_{b j}\right) \\
=\int_{\Sigma_{2}} \mathrm{~d} x^{1} \mathrm{~d} x^{2} \varepsilon^{c d} B_{\alpha \beta} \partial_{c} \phi^{\alpha} \partial_{d} \phi^{\beta},
\end{gathered}
$$

where

$$
B_{\alpha \beta}=\int_{H^{2}} d \operatorname{vol} \varepsilon^{i j}\left(\xi_{\alpha i}, \xi_{\beta j}\right)
$$

are components of the 2 -form $\mathbb{B}=\left(B_{\alpha \beta}\right)$ on the moduli space $\mathcal{M}=\Omega G$.

\section{REMARKS ON SUPERSTRINGS}

The adiabatic limit of supersymmetric Yang-Mills theories with a (partial) topological twisting on Euclidean manifold $\Sigma \times \tilde{\Sigma}$, where $\Sigma$ and $\tilde{\Sigma}$ are Riemann surfaces, was considered in [15]. Several sigma models with fermions on $\Sigma$ (including supersymmetric ones) were obtained. Switching to Lorentzian signature and adding constraints of type (18), which were not considered in [15], one can get stringy sigma-model resembling NSR strings. However, analysis of these sigma models demands more efforts and goes beyond the scope of our paper.

Another possibility is to consider ordinary Yang-Mills theory (11) but with Lie supergroup $G$ as the structure group. We restrict ourselves to the $N=2$ super translation group with ten-dimensional Minkowski space $\mathbb{R}^{9,1}$ as the bosonic part. This super translation group can be represented as the coset $[16,17]$

$$
G=\operatorname{SUSY}(N=2) / \operatorname{SO}(9,1),
$$

with coordinates $\left(X^{\alpha}, \theta^{A p}\right)$, where $\theta^{p}=\left(\theta^{A p}\right)$ are two Majorana-Weyl spinors in $d=10, \alpha=0, \ldots, 9, A=$ $1, \ldots, 32$ and $p=1,2$. The generators of $G$ obey the Lie superalgebra $\mathfrak{g}=\mathrm{Lie} G$,

$$
\begin{aligned}
\left\{\xi_{A p}, \xi_{B q}\right\} & =\left(\gamma^{\alpha} C\right)_{A B} \delta_{p q} \xi_{\alpha}, \quad\left[\xi_{\alpha}, \xi_{A p}\right]=0, \\
{\left[\xi_{\alpha}, \xi_{\beta}\right] } & =0,
\end{aligned}
$$

where $\gamma^{\alpha}$ are the $\gamma$ matrices in $\mathbb{R}^{9,1}$ and $C$ is the charge conjugation matrix. On the superalgebra $\mathfrak{g}$, we introduce the standard metric

$$
\left\langle\xi_{\alpha} \xi_{\beta}\right\rangle=\eta_{\alpha \beta}, \quad\left\langle\xi_{\alpha} \xi_{A p}\right\rangle=0 \quad \text { and } \quad\left\langle\xi_{A p} \xi_{B q}\right\rangle=0
$$


where $\left(\eta_{\alpha \beta}\right)=\operatorname{diag}(-1,1, \ldots, 1)$ is the Lorentzian metric on $\mathbb{R}^{9,1}$.

It was shown in [18] that the action functional for YangMills theory on $\Sigma_{2} \times H^{2}$ with the gauge group $G$, defined by (42)

$$
\begin{aligned}
S_{\varepsilon}= & \frac{1}{2 \pi} \int_{\Sigma_{2} \times H^{2}} \mathrm{~d}^{4} x \sqrt{\left|\operatorname{det} g_{\Sigma_{2}}\right|} \sqrt{\operatorname{det} g_{H_{2}}}\left\{\varepsilon^{2}\left\langle\mathcal{F}_{a b} \mathcal{F}^{a b}\right\rangle\right. \\
& \left.+2\left\langle\mathcal{F}_{a i} \mathcal{F}^{a i}\right\rangle+\varepsilon^{-2}\left\langle\mathcal{F}_{i j} \mathcal{F}^{i j}\right\rangle\right\}
\end{aligned}
$$

plus the Wess-Zumino-type term

$$
\begin{aligned}
S_{W Z}= & \frac{1}{\pi} \int_{\Sigma_{3} \times H^{2}} \mathrm{~d} x^{\hat{a}} \wedge \mathrm{d} x^{\hat{b}} \wedge \mathrm{d} x^{\hat{c}} \wedge \mathrm{d} x^{3} \\
& \wedge \mathrm{d} x^{4} f_{\Gamma \Delta \Lambda} \mathcal{F}_{\hat{a} i}^{\Gamma} \xi^{i} \mathcal{F}_{\hat{b} j}^{\Delta} \xi^{j} \mathcal{F}_{\hat{c} k}^{\Lambda} \xi^{k}
\end{aligned}
$$

yield the Green-Schwarz superstring action [17] in the adiabatic limit $\varepsilon \rightarrow 0$. Here $\Sigma_{3}$ is a Lorentzian manifold with the boundary $\Sigma_{2}=\partial \Sigma_{3}$ and local coordinates $x^{\hat{a}}$, $\hat{a}=0,1,2$; the structure constants $f_{\Gamma \Delta \Lambda}$ are given in [16] and $\left(\xi_{i}\right)=(\sin \varphi,-\cos \varphi)$ is the unit vector on $H^{2}$ running the boundary $S^{1}=\partial H^{2}$.

\section{SUPERSTRINGS FROM $d=3$ YANG-MILLS}

Here we will show that the Green-Schwarz superstrings with a world sheet $\Sigma_{2}$ can also be associated with a YangMills model on $\Sigma_{2} \times S^{1}$. When the radius of $S^{1}$ tends to zero, the action of this Yang-Mills model becomes the Green-Schwarz superstring action. So, we consider YangMills theory on a direct product manifold $M^{3}=\Sigma_{2} \times S^{1}$, where $\Sigma_{2}$ is a two-dimensional Lorentzian manifold discussed before and $S^{1}$ is the unit circle parametrized by $x^{3} \in$ $[0,2 \pi]$ with the metric tensor $g_{S^{1}}=\left(g_{33}\right)$ and $g_{33}=1$. As the structure group $G$ of Yang-Mills theory, we consider the super translation group in $d=10$ auxiliary dimensions (41) with the generators (42) and the metric (43) on the Lie superalgebra $\mathfrak{g}=\mathrm{Lie} G$. As in (20), we impose framing over $S^{1}$, i.e., consider the group of gauge transformations equal to the identity over $S^{1}$. Coordinates on $G$ are $X^{\alpha}$ and $\theta^{A p}$ introduced in the previous section. The 1-forms

$$
\Pi^{\Delta}=\left\{\Pi^{\alpha}, \Pi^{A p}\right\}=\left\{\mathrm{d} X^{\alpha}-\mathrm{i} \delta_{p q} \bar{\theta}^{p} \gamma^{\alpha} \mathrm{d} \theta^{q}, \mathrm{~d} \theta^{A p}\right\}
$$

form a basis of 1-forms on $G$ [16].

By using the adiabatic approach, we deform the metric on $\Sigma_{2} \times S^{1}$ and introduce

$$
\mathrm{d} s_{\varepsilon}^{2}=g_{\mu \nu}^{\varepsilon} \mathrm{d} x^{\mu} \mathrm{d} x^{\nu}=g_{a b} \mathrm{~d} x^{a} \mathrm{~d} x^{b}+\varepsilon^{2}\left(\mathrm{~d} x^{3}\right)^{2},
$$

where $\varepsilon \in[0,1]$ is a real parameter, $a, b=1,2$, $\mu, \nu=1,2,3$. This is equivalent to the consideration of the circle $S_{\varepsilon}^{1}$ of radius $\varepsilon$. It is assumed that for the fields $\mathcal{A}_{\mu}$ and $\mathcal{F}_{\mu \nu}$, there exist limits $\lim _{\varepsilon \rightarrow 0} \mathcal{A}_{\mu}$ and $\lim _{\varepsilon \rightarrow 0} \mathcal{F}_{\mu \nu}$. Indices are raised by $g_{\varepsilon}^{\mu \nu}$ and we have

$$
\begin{aligned}
& \mathcal{F}_{\varepsilon}^{a b}=g_{\varepsilon}^{a c} g_{\varepsilon}^{b d} \mathcal{F}_{c d}=\mathcal{F}^{a b}, \\
& \mathcal{F}_{\varepsilon}^{a 3}=g_{\varepsilon}^{a c} g_{\varepsilon}^{33} \mathcal{F}_{c 3}=\varepsilon^{-2} \mathcal{F}^{a 3},
\end{aligned}
$$

where indices in $\mathcal{F}^{\mu \nu}$ are raised by the nondeformed metric tensor.

We consider the Yang-Mills action of the form

$$
S_{\varepsilon}=\int_{M^{3}} \mathrm{~d}^{3} x \sqrt{\left|\operatorname{det} g_{\Sigma_{2}}\right|}\left\{\frac{\varepsilon^{2}}{2}\left\langle\mathcal{F}_{a b} \mathcal{F}^{a b}\right\rangle+\left\langle\mathcal{F}_{a 3} \mathcal{F}^{a 3}\right\rangle\right\},
$$

which for $\varepsilon=1$ coincides with the standard Yang-Mills action. Variations with respect to $\mathcal{A}_{\mu}$ and $g_{a b}$ yield the equations

$$
\begin{aligned}
\varepsilon^{2} D_{a} \mathcal{F}^{a b}+D_{3} \mathcal{F}^{3 b}=0, \quad D_{a} \mathcal{F}^{a 3}=0, \\
T_{a b}^{\varepsilon}=\varepsilon^{2}\left(g^{c d}\left\langle\mathcal{F}_{a c} \mathcal{F}^{b d}\right\rangle-\frac{1}{4} g_{a b}\left\langle\mathcal{F}_{c d} \mathcal{F}^{c d}\right\rangle\right) \\
+\left\langle\mathcal{F}_{a 3} \mathcal{F}_{b 3}\right\rangle-\frac{1}{2} g_{a b}\left\langle\mathcal{F}_{c 3} \mathcal{F}^{c 3}\right\rangle .
\end{aligned}
$$

In the adiabatic limit $\varepsilon \rightarrow 0$, Eqs. (50) and (51) become

$$
\begin{gathered}
D_{3} \mathcal{F}^{3 b}=0, \quad D_{a} \mathcal{F}^{a 3}=0, \\
T_{a b}^{0}=\left\langle\mathcal{F}_{a 3} \mathcal{F}_{b 3}\right\rangle-\frac{1}{2} g_{a b}\left\langle\mathcal{F}_{c 3} \mathcal{F}^{c 3}\right\rangle .
\end{gathered}
$$

Notice that as a function of $x^{3} \in S^{1}$, the field $\mathcal{A}_{3}$ belongs to the loop algebra $L \mathfrak{g}=\mathfrak{g} \oplus \Omega \mathfrak{g}$, where $\Omega \mathfrak{g}$ is the Lie superalgebra of the based loop group $\Omega G$. Let us denote by $\mathcal{A}_{3}^{0}$ the zero mode in the expansion of $\mathcal{A}_{3}$ in $\exp \left(\mathrm{i} x^{3}\right) \in S^{1}$ (Wilson line). The generic $\mathcal{A}_{3}$ can be represented in the form

$$
\mathcal{A}_{3}=h^{-1} \mathcal{A}_{3}^{0} h+h^{-1} \partial_{3} h,
$$

where the $G$-valued function $h$ depends on $x^{a}$ and $x^{3}$. For fixed $x^{a} \in \Sigma_{2}$, one can choose $h \in \Omega G=\operatorname{Map}\left(S^{1}, G\right) / G$. We denote by $\mathcal{N}$ the space of all $\mathcal{A}_{3}$ given by (54) and define the projection $\pi: \mathcal{N} \rightarrow G$ on the space $G$ parametrizing $\mathcal{A}_{3}^{0}$ since we want to keep only $\mathcal{A}_{3}^{0}$ in the limit $\varepsilon \rightarrow 0$. We denote by $Q$ the fibers of the projection $\pi$.

In the adiabatic approach, it is assumed that $\mathcal{A}_{3}^{0}$ depends on $x^{a} \in \Sigma_{2}$ only via the moduli parameters $\left(X^{\alpha}, \theta^{A p}\right) \in G$. Therefore, the moduli define the maps

$$
\left(X, \theta^{p}\right): \Sigma_{3} \rightarrow G
$$

which are not arbitrary; they are constrained by Eqs. (52) and (53). The derivatives $\partial_{a} \mathcal{A}_{3}$ of $\mathcal{A}_{3} \in \mathcal{N}$ belong to the tangent space $T_{\mathcal{A}_{3}} \mathcal{N}$ of the space $\mathcal{N}$. Using the projection $\pi: \mathcal{N} \rightarrow G$, one can decompose $\partial_{a} \mathcal{A}_{3}$ into two parts, 


$$
T_{\mathcal{A}_{3}} \mathcal{N}=\pi^{*} T_{\mathcal{A}_{3}^{0}} G \oplus T_{\mathcal{A}_{3}} Q \Leftrightarrow \partial_{a} \mathcal{A}_{3}=\Pi_{a}^{\Delta} \xi_{\Delta 3}+D_{3} \epsilon_{a},
$$

where $\Delta=(\alpha, A p)$ and

$$
\Pi_{a}^{\alpha}:=\partial_{a} X^{\alpha}-\mathrm{i} \delta_{p q} \bar{\theta}^{p} \gamma^{\alpha} \partial_{a} \theta^{q}, \quad \Pi_{a}^{A p}:=\partial_{a} \theta^{A p} .
$$

In (56), $\epsilon_{a}$ are $\mathfrak{g}$-valued parameters $\left(D_{3} \epsilon_{a} \in T_{\mathcal{A}_{3}} Q\right)$ and the vector fields $\xi_{\Delta 3}$ on $G$ can be identified with the generators $\xi_{\Delta}=\left(\xi_{\alpha}, \xi_{A p}\right)$ of $G$.

On $\xi_{\Delta 3}$ we impose the gauge-fixing condition

$$
D_{3} \xi_{\Delta 3}=0 \stackrel{(56)}{\Rightarrow} D_{3} D_{3} \epsilon_{a}=D_{3} \partial_{a} \mathcal{A}_{3} .
$$

Recall that $\mathcal{A}_{3}$ is fixed by (54) and $\mathcal{A}_{a}$ are yet free. In the adiabatic approach, one chooses $\mathcal{A}_{a}=\epsilon_{a}$ (cf., [5,6]) and obtains

$$
\mathcal{F}_{a 3}=\partial_{a} \mathcal{A}_{3}-D_{3} \mathcal{A}_{a}=\Pi_{a}^{\Delta} \xi_{\Delta 3} \in T_{\mathcal{A}_{3}^{0}} G .
$$

Substituting (59) into the first equation in (52), we see that they are resolved due to (58). Substituting (59) into the action $S_{0}=\lim _{\varepsilon \rightarrow 0} S_{\varepsilon}$ given by (49) and integrating over $x^{3}$, we obtain the effective action

$$
S_{0}=2 \pi \int_{\Sigma_{2}} \mathrm{~d}^{2} x \sqrt{\left|\operatorname{det} g_{\Sigma_{2}}\right|} \mid g^{a b} \Pi_{a}^{\alpha} \Pi_{b}^{\beta} \eta_{\alpha \beta},
$$

which coincides with the kinetic part of the Green-Schwarz superstring action [17]. One can show (cf., [14]) that the second equations in (52) are equivalent to the EulerLagrange equations for $\left(X^{\alpha}, \theta^{A p}\right)$ following from (60). Finally, substituting (59) into (53), we obtain the equations

$$
\Pi_{a}^{\alpha} \Pi_{b}^{\beta} \eta_{\alpha \beta}-\frac{1}{2} g_{a b} g^{c d} \Pi_{c}^{\alpha} \Pi_{d}^{\beta} \eta_{\alpha \beta}=0,
$$

which can also be obtained from (60) by variation of $g^{a b}$.

For getting the full Green-Schwarz superstring action one should add to (60) a Wess-Zumino-type term which is described as follows [16,17]. One should consider a Lorentzian 3-manifold $\Sigma_{3}$ with the boundary $\Sigma_{2}=\partial \Sigma_{3}$ and coordinates $x^{\hat{a}}, \hat{a}=0,1,2$. On $\Sigma_{3}$, one introduces the 3 -form [16]

$$
\Omega_{3}=\mathrm{id} x^{\hat{a}} \prod_{\hat{a}}^{\alpha} \wedge\left(\check{\mathrm{d}} \bar{\theta}^{1} \gamma^{\beta} \wedge \check{\mathrm{d}} \theta^{1}-\breve{\mathrm{d}} \bar{\theta}^{2} \gamma^{\beta} \wedge \check{\mathrm{d}} \theta^{2}\right) \eta_{\alpha \beta}=\breve{\mathrm{d}} \Omega_{2},
$$

where

$$
\begin{aligned}
\Omega_{2} & =-\mathrm{i} \check{\mathrm{d}} X^{\alpha} \wedge\left(\bar{\theta}^{1} \gamma^{\beta} \mathrm{d} \theta^{1}-\bar{\theta}^{2} \gamma^{\beta} \mathrm{d} \theta^{2}\right) \quad \text { with } \\
\check{\mathrm{d}} & =\mathrm{d} x^{\hat{a}} \frac{\partial}{\partial x^{\hat{a}}} .
\end{aligned}
$$

Then the term

$$
S_{\mathrm{WZ}}=\int_{\Sigma_{3}} \Omega_{3}=\int_{\Sigma_{2}} \Omega_{2}
$$

is added to (60) with a proper coefficient $\varkappa$ and $S_{\mathrm{GS}}=$ $S_{0}+\varkappa S_{\mathrm{WZ}}$ is the Green-Schwarz action for the superstrings of type I, IIA, and IIB.

To get (63) from Yang-Mills theory, we consider the manifold $\Sigma_{3} \times S^{1}$ and notice that in addition to (59) we now have the components

$$
\begin{aligned}
\mathcal{F}_{03} & =\Pi_{0}^{\Delta} \xi_{\Delta 3} \\
& =\left(\partial_{0} X^{\alpha}-\mathrm{i} \delta_{p q} \bar{\theta}^{p} \gamma^{\alpha} \partial_{0} \theta^{q}\right) \xi_{\alpha 3}+\left(\partial_{0} \theta^{A p}\right) \xi_{A p 3} .
\end{aligned}
$$

We introduce 1-forms $F_{3}:=\mathcal{F}_{\hat{a} 3} \mathrm{~d} x^{\hat{a}}$ on $\Sigma_{3}$, where $\mathcal{F}_{\hat{a} 3}(\varepsilon)$ are general Yang-Mills fields on $\Sigma_{3} \times S^{1}$ which take the form (59), (64) only in the limit $\varepsilon \rightarrow 0$, and consider the functional

$$
S_{\mathrm{WZ}}^{\mathrm{YM}}=\int_{\Sigma_{3} \times S^{1}} f_{\Delta \Lambda \Gamma} F_{3}^{\Delta} \wedge F_{3}^{\Lambda} \wedge F_{3}^{\Gamma} \wedge \mathrm{d} x^{3},
$$

where the explicit form of the constant $f_{\Delta \Lambda \Gamma}$ can be found in [16]. Therefore, the Yang-Mills action (49) plus (65) in the adiabatic limit $\varepsilon \rightarrow 0$ becomes the GreenSchwarz action. This result can be considered as a generalization of the Green result [19] who derived the superstring theory in a fixed gauge from Chern-Simons theory on $\Sigma_{2} \times \mathbb{R}$.

\section{CONCLUDING REMARKS}

We have shown that bosonic strings and GreenSchwarz superstrings can be obtained via the adiabatic limit of Yang-Mills theory on manifolds $\Sigma_{2} \times H^{2}$ with a Wess-Zumino-type term. Notice that the constraint equations (15) on the Yang-Mills energy momentum tensor with $\varepsilon>0$ are important for restoring the unitarity of Yang-Mills theory on $\Sigma_{2} \times H^{2}$. More interestingly, the same result is also obtained by considering Yang-Mills theory on three-dimensional manifolds $\Sigma_{2} \times S_{\varepsilon}^{1}$ with the radius of the circle $S_{\varepsilon}^{1}$ given by $\varepsilon \in[0,1]$. For $\varepsilon \neq 0$ we have well-defined quantum Yang-Mills theory on $\Sigma_{2} \times S_{\varepsilon}^{1}$. For $\varepsilon \rightarrow 0$ we get superstring theories. This raises hopes that various results for superstring theories can be obtained from results of the associated Yang-Mills theory on $\Sigma_{2} \times S_{\varepsilon}^{1}$.

\section{ACKNOWLEDGMENTS}

This work was partially supported by the Deutsche Forschungsgemeinschaft Grant No. LE 838/13. 
[1] M. B. Green, J. H. Schwarz, and E. Witten, Superstring Theory: Volumes 1 \& 2 (Cambridge University Press, Cambridge, England, 1987).

[2] J. Polchinski, String Theory: Volumes 1 \& 2 (Cambridge University Press, Cambridge, England, 1998).

[3] K. Becker, M. Becker, and J. H. Schwarz, String Theory and M-Theory: A Modern Introduction (Cambridge University Press, Cambridge, England, 2007).

[4] D. Bailin and A. Love, Supersymmetric Gauge Theory and String Theory (Taylor \& Francis, London, 1994).

[5] N.S. Manton and P. Sutcliffe, Topological Solitons (Cambridge University Press, Cambridge, England, 2004).

[6] E. J. Weinberg and P. Yi, Magnetic monopole dynamics, supersymmetry, and duality, Phys. Rep. 438, 65 (2007).

[7] M. Shifman, Advanced Topics in Quantum Field Theory (Cambridge University Press, Cambridge, England, 2012).

[8] S. Dostoglou and D. A. Salamon, Self-dual instantons and holomorphic curves, Ann. Math. 139, 581 (1994).

[9] D. A. Salamon, Notes on flat connections and the loop group, University of Warwick, 1998.

[10] S. K. Donaldson, Boundary value problems for Yang-Mills fields, J. Geom. Phys. 8, 89 (1992).
[11] S. Jarvis and P. Norbury, Degenerating metrics and instantons on the four-sphere, J. Geom. Phys. 27, 79 (1998).

[12] A. N. Pressley and G. B. Segal, Loop Groups (Oxford University Press, New York, 1984).

[13] J. M. Figueroa-O'Farrill, C. Kohl, and B. J. Spence, Supersymmetric Yang-Mills, octonionic instantons and triholomorphic curves, Nucl. Phys. B521, 419 (1998).

[14] A. D. Popov, Loop groups in Yang-Mills theory, Phys. Lett. B 748, 439 (2015).

[15] M. Bershadsky, A. Johansen, V. Sadov, and C. Vafa, Topological reduction of 4D SYM to $2 \mathrm{D} \sigma$-models, Nucl. Phys. B448, 166 (1995).

[16] M. Henneaux and L. Mezincescu, A $\sigma$-model interpretation of Green-Schwarz covariant superstring action, Phys. Lett. 152B, 340 (1985).

[17] M. B. Green and J. H. Schwarz, Covariant description of superstrings, Phys. Lett. 136B, 367 (1984).

[18] A. D. Popov, Green-Schwarz superstring as subsector of Yang-Mills theory, arXiv:1506.02175.

[19] M. B. Green, Supertranslations, superstrings and ChernSimons forms, Phys. Lett. B 223, 157 (1989). 